Provided for non-commercial research and education use. Not for reproduction, distribution or commercial use.

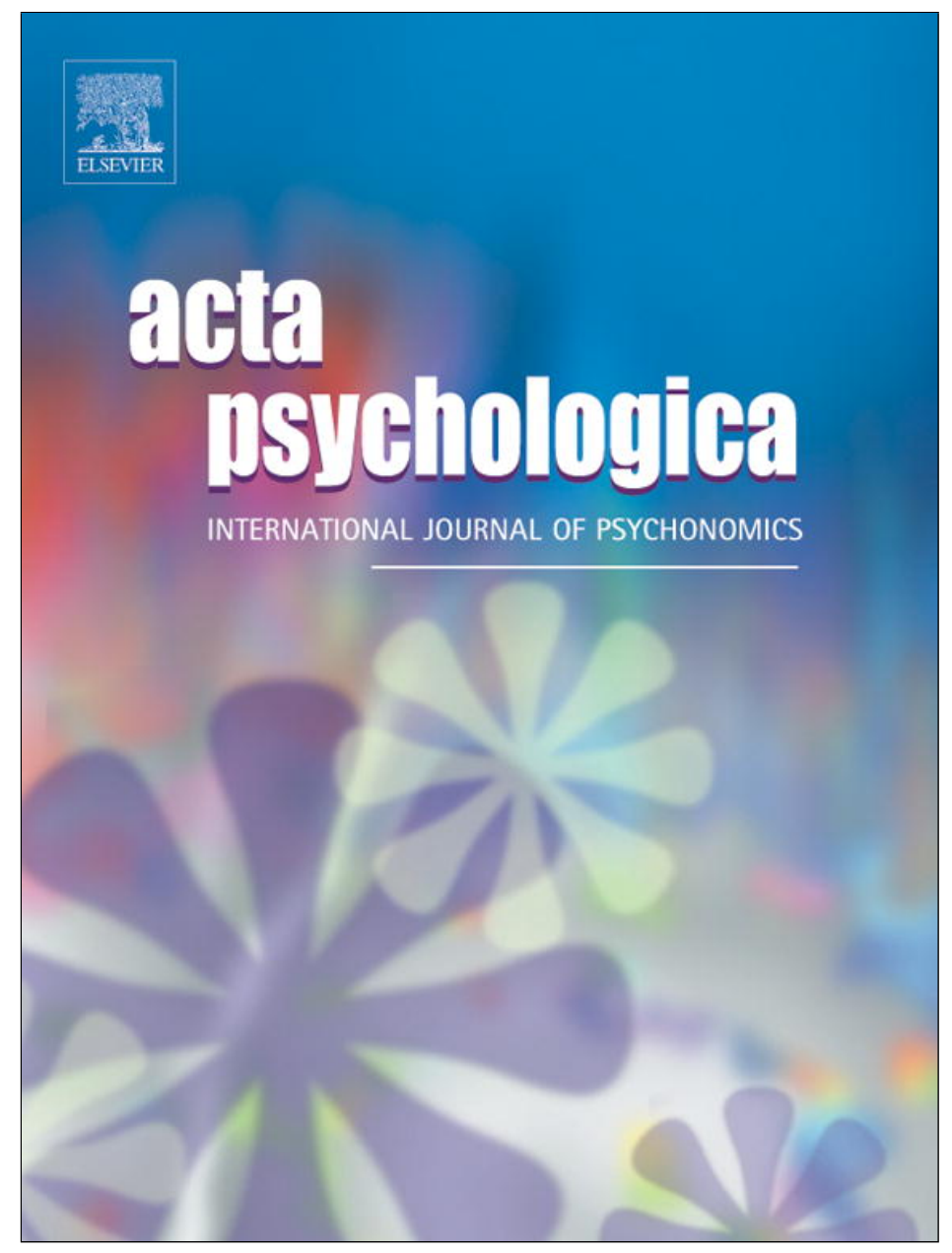

(This is a sample cover image for this issue. The actual cover is not yet available at this time.)

This article appeared in a journal published by Elsevier. The attached copy is furnished to the author for internal non-commercial research and education use, including for instruction at the authors institution and sharing with colleagues.

Other uses, including reproduction and distribution, or selling or licensing copies, or posting to personal, institutional or third party websites are prohibited.

In most cases authors are permitted to post their version of the article (e.g. in Word or Tex form) to their personal website or institutional repository. Authors requiring further information regarding Elsevier's archiving and manuscript policies are encouraged to visit:

http://www.elsevier.com/copyright 


\title{
Individual differences in cognitive representations of action influence the activation of goal concepts
}

\author{
S. Belayachi ${ }^{\mathrm{a}, *}$, M. Van der Linden ${ }^{\mathrm{a}, \mathrm{b}}$ \\ a Cognitive Psychopathology Unit, University of Liège, Belgium \\ ${ }^{\mathrm{b}}$ Cognitive Psychopathology and Neuropsychology Unit, University of Geneva, Switzerland
}

\section{A R T I C L E I N F O}

\section{Article history:}

Received 24 April 2012

Received in revised form 28 November 2012

Accepted 20 December 2012

Available online $\mathrm{xxxx}$

\section{PsycINFO classification:}

2340

3120

Keywords:

Action identification

Action processing

Individual differences

Automatic goal activation

\begin{abstract}
A B S T R A C T
Goal representations play a key role in various psychological processes, including behavioral regulation, self-perception and social understanding. Research on cognitive representations of action has identified individual differences in the general tendency to construe actions in terms of their goal (vs. movement parameters), which can be reliably assessed with the Behavior Identification Form (BIF). The aim of the present study was to examine how individual differences in action identification, as measured by the BIF, affect online processing of action in a laboratory study. The main results showed that the level of action identification predicted participants' performance in a task designed to implicitly assess people's automatic processing of action regarding goal features. We discussed the possible role of impaired goal processing in psychological dysfunctions.
\end{abstract}

(c) 2013 Elsevier B.V. All rights reserved.

\section{Introduction}

Mental representations of self or other behaviors are not just progressive reconstructions of what one is doing in the current situation; the way people perceive and understand what they are doing (or what others do) may also depend on how pre-existing mental representations of actions are used for action processing. This kind of interconnection between action and cognition has been intensively explored in the context of action identification theory (Vallacher \& Wegner, 1985, 1987). Action identification theory posits that any behavior can be identified within a cognitive hierarchy of meanings: the higher-level meanings relate to the pursued goal and anticipated outcomes; the lower-level meanings, however, represent instrumental features and movement parameters (Vallacher \& Wegner, 1985, 1987, 1989). Vallacher and Wegner suggested that the level at which an action is identified may reflect the particular representation (movement parameters vs. abstract goal) on the basis of which a performed action is monitored and a perceived action is understood. For example, identifying the act of "locking the door" as "putting a key in the lock" shows that the instrumental representation is most accessible, whereas identifying this act as "securing the house" reflects the predominant accessibility of the goal representation.

\footnotetext{
* Corresponding author at: Cognitive Psychopathology Unit, Department of Cognitive Sciences, University of Liège, Belgium. Tel.: + 32436659 79; fax: + 3243662808 .

E-mail address: sanaa.belayachi.ulg@gmail.com (S. Belayachi).
}

When an act can be identified at both high and low levels (as in the case of habitual actions), people adopt higher (more meaningful) levels of action identification over lower-level ones (Vallacher \& Wegner, 1987); however, whenever an act becomes complex to process (e.g., disruption, errors), people tend to adopt lower levels.

Nevertheless, the research on action identification has shown not only that there are individual differences in the ability to process familiar actions with a view to goal features, but also that such individual differences in action identification may promote different modes of action processing. Vallacher and Wegner developed a reliable tool for assessing individual differences in action identification, the Behavior Identification Form (BIF). By using this instrument, Vallacher and Wegner (1989) showed that people tend to process action based upon a preferential level of action identification. Thus, there are people who generally process action with abstract features in mind, that is, regarding the purpose and implications of action (high-level agents), while other people tend to identify actions at a uniformly low level, that is, based on procedural aspects and motor subcomponents (low-level agents). High-level agents are characterized by a good ability to easily adopt an appropriate level of identification for different types of actions, while low-level agents may have more difficulties in adapting representations to action constraints. In the specific case of familiar actions, people who have a general tendency to systematically adopt low-level identities seem to find it difficult in maintaining their actions or understanding perceived actions with goal representations in mind. This kind of difficulty can be assessed 
using the BIF, an instrument designed by Vallacher and Wegner (1989) to measure individual differences in action identification level across an array of routine actions. Each item of the BIF consists of an action followed by two alternatives or "identities," one of which is lower (i.e., movement-focused description of action) and one higher in level (i.e., goal focused). For example, the act of "locking the door" is followed by (a) "putting a key in the lock" (low-level action identification) and (b) "securing the house" (high-level action identification). For each action, participants must choose the alternative that best describes the action for them. The number of high-level identities chosen defines the subjects' level of action identification.

By using the BIF, Vallacher and Wegner showed that the preferential level of action identification is related to distinct modes of action processing. Because performing habitual actions with unavailable or irrelevant goal representations may simply disorganize the action flow, people characterized by a low level of action identification, that is, mainly focused on the movement parameters and mechanistic aspects of action, are more prone to ineffectiveness in everyday actions (e.g., emergence of new courses of action, more attention focused on details of performance, doubts about whether the action has been completed; Vallacher \& Wegner, 1989). They may also have difficulties in understanding events regarding goal features (e.g., Belayachi \& Van der Linden, 2009). By contrast, by focusing on the abstract goals and implications of behaviors, people with a high level of action identification tend to have greater overall efficiency in everyday actions (e.g., less disruption, fewer resources needed and better goal completion in everyday behaviors) and have greater facility in perceiving other's intention and goal.

It is generally acknowledged that humans are spontaneously attuned to a high-level understanding of events and behaviors (e.g., Csibra, Gergely, Biro, Koos, \& Brockbank, 1999; Hassin, Aarts, \& Ferguson, 2005; Heider \& Simmel, 1944; McKoon \& Ratcliff, 1992; Vallacher \& Wegner, 1987; Zacks, 2004). However, there are low- and high-level agents, who may differ in their ability to understand behavior in terms of its underlying goal. One possibility is that the level of action identification may be related to a more basic cognitive process responsible for an automatic orientation towards comprehensive understanding of actions. Even though people can consciously activate or build goal representations (e.g., through verbalization, conscious inferences or conscious effort to remember a goal), we assume that, in everyday behavioral situations, the limited resources of the cognitive system do not allow them to systematically process goal-related features at a conscious level (i.e., for behavioral regulation or for understanding others' actions). Thus, it is possible that people with a chronic low level of action identification may be characterized by an impairment of the automatic activation or accessibility of goal representations. It is often suggested that people with a chronic low level of action identification may be characterized by an undermined ability to activate or keep goal representations in mind during action processing (Belayachi \& Van der Linden, 2009; Vallacher \& Wegner, 1989; Wegner, Vallacher, Macomber, Wood, \& Arps, 1984).

To sum up, although there are various ways of construing and regulating one's action or understanding that of others', people seem to identify their actions based on a preferential level, which reflects the internal representation (goal vs. movements) that is generally activated during action processing. Vallacher and Wegner developed a reliable tool for assessing such individual differences in basic action processing, the BIF, which has been related to different modes of action processing in everyday behaviors. The current study aimed to examine how the level of action identification affects action processing in a laboratory experiment designed to assess the automaticity of goal activation during action processing. According to Vallacher and Wegner, people with a chronic low level of action identification may be characterized by difficulties activating or accessing goal representations during action processing. This assumption will be tested by more directly examining the extent to which the difference between low- and high-level agents can be construed in terms of differential degrees of automatic activation of goal representations during action processing.

This issue can be examined by using a task designed to assess automatic goal inferences. In this task, inspired by Hassin et al. (2005), participants read short sentences after which they perform a lexical decision task; that is, they see a string of letters (test word) for which they must rapidly determine whether it is a word or not. There are two kinds of experimental sentences: those that imply a goal and those that do not. The test word, in both cases, is a goal that is not explicitly mentioned in the sentence. The authors suggested that, if goals are automatically inferred, then their accessibility should increase after reading goal-implying sentences, thus making the judgment task easier. Consistently with this assumption, Hassin et al. (2005) demonstrated such a goal effect by observing that responses after goal-implying sentences were faster than responses after control sentences. If the level of action identification is related to the degree of accessibility of goal representations, then we could expect that the level of agency would be related to the strength of the goal effect on response latencies.

\section{Method}

\subsection{Participants}

Seventy-seven undergraduates ( 32 males and 45 females) from the University of Liège, aged between 18 and 30, took part in the study. Participants were randomly recruited from various faculties and schools of the university; they were not compensated for their participation. Their mean age was 21.81 years $(S D=2.26$ years).

\subsection{Apparatus and measures}

The goal activation task was programmed in the Matlab environment; we used the same task administration and scoring methods described in Hassin et al. (2005). In this task, a series of situations were presented one by one to participants, who, after each situation, had to perform a lexical decision task. These situations are sentences depicting basic behavioral contexts. There are two types of experimental situations: situations involving a specific goal (e.g., The woman connects the garden hose and walks towards the car; the goal=washing the car) and control situations, which contained the same words as situations involving a goal but associated in a different way so that they activate a different semantic field (e.g., the woman walks to the car and throws the garden hose into it). Each situation was immediately followed by a lexical decision task, which involved presenting strings of letters for which participants had to rapidly determine whether they were words or not. In fact, all the experimental situations were systematically followed by the presentation of a real word. These test words represent the specific goal that is implicitly indicated in each goal-implying situation (e.g., wash). Words presented in the lexical decision tasks following goal-implying sentences and their corresponding control situations are the same. We expected, as demonstrated by Hassin et al. (2005), that reaction times for test words would be faster when preceded by goal-implying sentences than when preceded by control situations. In order to prevent participants from guessing the real purpose of the task, the task also contained distractor situations that described an action (e.g., the woman puts the pie in the car and goes to the party; the woman locks the door and puts the key in her purse). Lexical decision tasks following each of these distractor situations consisted in presenting either a word (unrelated to any of the situations presented in this study) or a non-word.

In total, there were 7 situations involving a goal, 7 control situations that had the same semantic context but referred to a different goal and 74 distractor situations. Each sentence remained on the screen for $3 \mathrm{~s}$, and then a row of asterisks appeared for $500 \mathrm{~ms}$, followed by a word 
or a non-word (i.e., lexical decision task). Participants had to press a left-hand key for letter strings recognized as words and a right-hand key for non-words. All the 14 experimental situations were followed by a lexical decision task on a word (i.e., specific goal); 30 of the distractor situations were also followed by a word, while 44 distractor situations were followed by a non-word. The lexical decision task was not limited in time; the reaction times and the number of correct responses were computed for each test word of the lexical decision task. Finally, the interstimulus interval was $2 \mathrm{~s}$ after a participant responded. Items were presented in two parts, separated by a 1 -minute break. The first part of the task consisted in presenting either the 7 situations involving a goal or the 7 control situations, in addition to 37 distractor situation conditions ( 15 followed by a word and 22 followed by a non-word in the lexical decision task); in the second part, the 7 remaining experimental situations (the control or the goal situations) and the other half of the distractor situations were presented. For each part of the task, the order of item presentation was randomly determined at the beginning of the task. Two versions of the task were created: in the first version (goal/control), goal-implying sentences were presented first (in part 1); in the second version (control/goal), situations involving a goal were presented after the control situations (in the second part of the task). This configuration allows one to avoid certain problems related to the many similarities between the two types of sentences. Indeed, because the lexemic contents of the goal-implying sentences and their corresponding control situations are so similar, participants could be aware of the similarities if these sentences are presented close together in time. Participants might also notice that the same goal word is presented twice, in sentences containing the same words. To prevent such problems, the goal-implying sentences and their corresponding control situations were presented in two separate blocks.

\subsubsection{Response latency}

Average response latency was calculated for the test words that followed the goal-implying sentences and the control sentences. Incorrect responses on test words (i.e., words erroneously recognized as non-words) were excluded from the analyses (there were $1.5 \%$ of incorrect responses in the control condition and $0.6 \%$ of incorrect responses in the goal condition). Response latencies that deviated more than 3 SDs from the overall mean were also excluded from the analyses. We then computed the average reaction time for both the goal condition $\left(\mathrm{RT}_{\text {goal }}\right)$ and the control condition $\left(\mathrm{RT}_{\text {control }}\right)$.

\subsubsection{Goal effect measure}

We also used the difference in RTs between the two conditions to quantify the amount of the decrease in response latency due to the prior goal-implying sentences (i.e., the overall effect of goal). For each participant, we computed a proportional index, defined as the percentage difference between the participant's mean RT in the goal condition and the participant's mean RT in the control condition (i.e., $\left[\mathrm{RT}_{\text {goal }}-\mathrm{RT}_{\text {control }}\right] / \mathrm{RT}_{\text {control }} * 100$ ). Values for the proportional index ranged from -34.62 to 18.96 .

\subsubsection{BIF}

Participants completed the French version of the BIF (Vallacher \& Wegner, 1989). The BIF is a one-dimensional scale designed to measure individual differences in action identification level (i.e., level of personal agency) across an array of routine actions. Each item of the BIF consists of an action followed by two alternatives or "identities," one of which is lower (i.e., movement-focused description of action) and one higher in level (i.e., goal-focused). For each action, participants must choose the alternative that best describes the action for them. The number of high-level identities chosen defines the subjects' level of action identification. In a recent replication study (Belayachi \& der Linden, submitted for publication), the psychometric properties of the 23-item French version of the BIF have been found to be comparable to those of the original version (Vallacher \& Wegner, 1989). In the present study, the scale was shown to be reliable, with a Cronbach's alpha of .91 .

\subsection{Design}

All participants were presented with both types of experimental situations (goal-implying and control sentences). Hence, the type of sentence (control vs. goal) constituted a within-participant factor. To control for order effects, half of the participants were given the goal/control version, and the other half received the control/goal order. Order (control/goal vs. goal/control) therefore constituted a between-participant factor. As well, participants' level of action identification (high-level vs. low-level) was measured with the BIF and used as a between-participant factor.

\subsection{Procedure}

Informed consent was obtained from all participants following a full explanation of the experimental procedure. Detailed written and oral instructions explained that they would participate in a study of language comprehension. Participants were told that they would be shown letter strings on the screen and that we were interested in their ability to determine whether these sets of letters were words or not. They were further informed that we wished to assess whether their ability to make such judgments would be facilitated or impaired by the previous presentation of verbal material. Participants performed the experimental task and completed the BIF (as well as other questionnaires unrelated to the present study) in an individual testing session; the order of administration of the task and the questionnaires was counterbalanced across participants. Finally, participants were debriefed after the completion of the entire protocol. This debriefing revealed that none of the participants realized the true nature of the task or consciously inferred goals from the situations.

\section{Results}

Table 1 summarizes the sample's mean scores on the BIF, the goal effect index and the average response latency for the test words that followed the seven goal-implying sentences (RT-goal) and the seven control sentences (RT-control).

\subsection{Group comparisons}

To directly address the question of whether low-level agents are characterized by a problem affecting automatic goal activation, compared to high-level agents, we divided the overall sample into two groups - high on level of action identification (high-level agents, $n=42$ ) and low on level of action identification (Low-level agents, $n=35)$ - on the basis of the median sample value for the BIF measure $(M d n=15)$. The mean BIF score was $11.26(S D=2.67)$ in the low-level agent group and $17.83(S D=2.26)$ in the high-level agent

\section{Table 1}

Mean scores and SDs for the action identification scores, RTs in the goal and control conditions, goal effect measure in the overall sample and Pearson correlations between action identification and the three measures of the task.

\begin{tabular}{|c|c|c|c|c|}
\hline & \multirow[t]{2}{*}{ Mean } & \multirow[t]{2}{*}{$S D$} & \multicolumn{2}{|c|}{$\begin{array}{l}\text { Correlation with the } \\
\text { BIF }\end{array}$} \\
\hline & & & $r$ & $p$ \\
\hline BIF score & 14.84 & 4.10 & / & I \\
\hline $\mathrm{RT}_{\text {control }}$ & 705 & 174 & 0.11 & 0.350 \\
\hline $\mathrm{RT}_{\text {goal }}$ & 644 & 169 & -0.05 & 0.647 \\
\hline Goal effect measure & -8.01 & 11.65 & -0.31 & 0.007 \\
\hline
\end{tabular}


Table 2

Mean scores and SDs for the action identification scores, RTs in the goal and control conditions and the difference between these two conditions for each action identification group.

\begin{tabular}{lll}
\hline & High-level agents $\mathrm{n}=42$ & Low level agents $\mathrm{n}=35$ \\
\hline Age & $22(2.40)$ & $21.57(2.09)$ \\
Gender & & \\
$M$ & 21 & 11 \\
$F$ & 21 & 24 \\
BIF score & $17.83(2.26)$ & $11.26(2.67)$ \\
$\mathrm{RT}_{\text {control }}$ & $700(165)$ & $712(186)$ \\
$\mathrm{RT}_{\text {goal }}$ & $618(137)$ & $676(199)$ \\
Goal effect measure & $-10.61(11.01)$ & $-4.89(11.80)$ \\
\hline
\end{tabular}

group (see Table 2 for further details). The dependent measure of interest was the average response latency for the test words that followed the seven goal-implying sentences (RTgoal) and the seven control sentences (RTcontrol).

In order to control for the order effect, the RTs were entered in a 2 (type of sentence: control vs. goal - within-participants factor $) \times 2$ (order: control/goal vs. goal/control) $\times 2$ (group: high-level agents vs. low-level agents - between-participants factors) ANOVA. This analysis yielded a statistically significant main effect of type of sentence, $F(1$, $73)=32.14, p<.001\left(\eta^{2} p=0.31\right)$, suggesting that responses to the test words were significantly faster when preceded by goal-implying sentences than when preceded by control sentences. There was also a main effect of order, $F(1,73)=5.14, p=.026\left(\eta^{2} \mathrm{p}=0.07\right)$, suggesting that participants who received the goal sentences first were generally faster $(M=636 ; S D=26)$ than those who received the control sentences first $(M=717 ; S D=25)$, although there were no interactions between order and type of sentence $[F(1,73)=.04, p=.849]$ or between order and group $[F(1,73)=.71, p=.401]$. Finally, there was no main effect of group, $F(1,73)=.55, p=.461$, while the interaction between type of sentence and group was found to be statistically significant, $F(1,73)=4.49, p=.037\left(\eta^{2} \mathrm{p}=0.06\right)$ (see Fig. 1$)$. There was no triple interaction, $F(1,73)=1.58, p=.213$. Paired t-tests performed separately for each group on RTs across the two conditions (goal vs. control) revealed that RTs were shorter in the goal condition than in the control condition for high-level agents, $t(41)=-5.67, p<.001$ (Cohen's $\mathrm{d}=-.87$ ) and, to a lesser extent, for low-level agents as well, $t(34)=-2.37, p=.024$ (Cohen's $d=-.40$ ). We then performed t-tests on RTs in the control and goal conditions and on the goal effect measure (i.e., the proportional index: the percentage difference between the mean RT in the goal condition and the mean RT in the

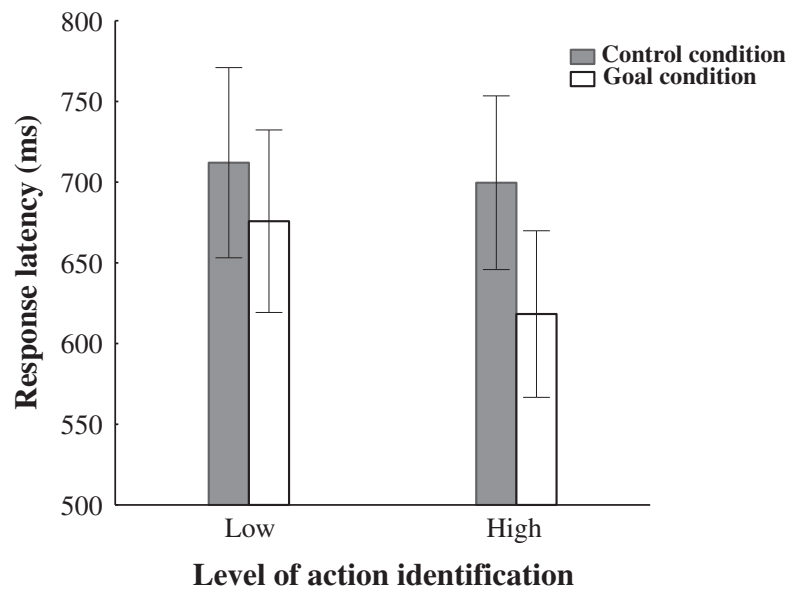

Fig. 1. Response latency as function of the type of sentence (control vs. goal), for each group (low-level vs. high-level of action identification). Error bars represent standard errors. control condition) across the two groups. These analyses showed that there were no group differences in RTs in the control condition, $t(75)=-0.31, p=.757$, or the goal condition, $t(75)=-1.50, p=$ .139; however, high-level agents had a higher overall benefit of goal than low-level agents on the proportional index, $t(75)=-2.19$, $p=.031$ (Cohen's $\mathrm{d}=.50$ ).

\subsection{Correlation analyses}

We then explored the association between measures on the task and level of action identification. Table 1 summarizes the correlations between the level of action identification and the three measures of the task (i.e., RTs in the goal and control conditions and the goal effect index). These analyses revealed that there were no correlations between BIF scores and RTs in the goal and control conditions. However, there was a statistically significant association between the level of action identification and the overall effect of goal (as measured by proportional indexes), suggesting that a higher level of action identification is related to a greater difference between RTgoal and RTcontrol (see Fig. 2).

\section{Discussion}

This article examines the relationship between the BIF, which assesses individual differences in action identification, and on-line processing of action in a laboratory experiment. The results can be summarized as follows. First, the results suggested that the French version of the BIF is a reliable instrument with psychometric properties similar to those observed in the original version (Vallacher \& Wegner, 1989). In addition, scores on the BIF were normally distributed, which supports the idea that the preferential level of action identification is distributed along a continuum ranging from extremely concrete (i.e., mainly based on mechanistic aspects and movement parameters) to extremely abstract (i.e., predominantly based on goal-related features). In addition, there was no gender effect on the level of action identification.

More importantly, we used a lexical decision task, in which the test words were goal concepts that had not appeared in the target sentences. In line with previous findings (Hassin et al., 2005), the results show that participants' judgments in the lexical decision task came more easily after goal-implying scenarios than after control scenarios (i.e., RTs for test words were faster when preceded by goalimplying sentences than when preceded by control situations). This may be due to the fact that automatic online inferences make goal representations more accessible. As expected, scores on the BIF

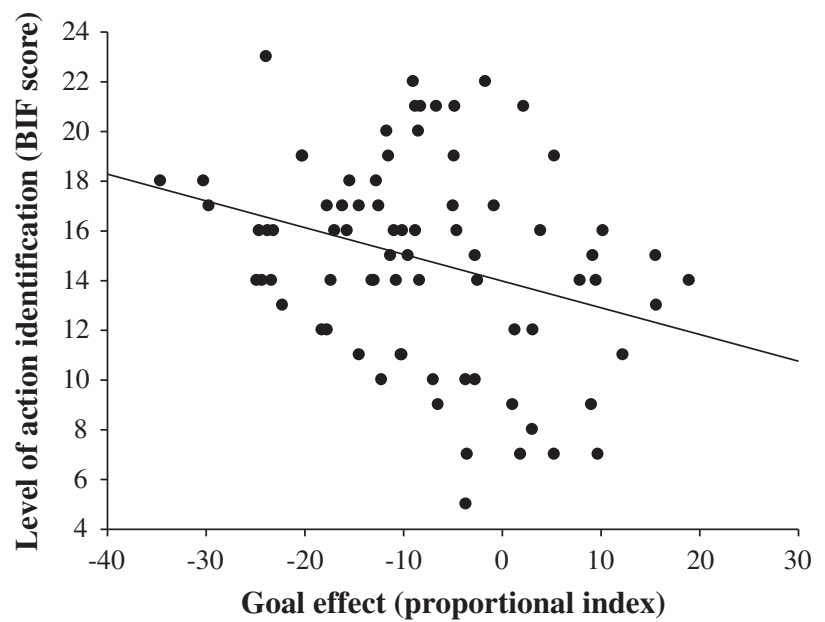

Fig. 2. The relationship between the level of action identification (i.e., BIF scores) and the overall benefit of goal (as measured by proportional indexes). 
were found to be correlated with a greater goal effect (i.e., the amount of difference in reaction time from the control condition to the goal condition).

When we compared participants with a high level of action identification to those with a low level of action identification, we found a statistically significant interaction between the level of action identification and the type of sentence preceding the test word. Further analyses revealed that there were no differences between the two groups in terms of participants' RTs in the control condition or in the goal condition; however, the overall effect of goal (i.e., goal effect index) was significantly higher for the high-level group than for the low-level group. These results suggest that goal-implying sentences enhanced the speed of processing of the subsequent test word (i.e., explicit goal) more markedly among participants with a preferential high level of action identification than in participants from the low-level group.

By and large, our results are consistent with Vallacher and Wegner's conceptualization of action identification levels and their suggestion that individual differences in action identification may imply different modes of action processing. Our results support the idea that level of action identification is related to the automatic activation of goal representations during action processing. Indeed, high-level agents may appear to activate goal representations faster when processing verbal materials related to actions than their low-level counterparts. This could suggest that, compared to high-level agents, low-level agents are characterized by increased difficulty in maintaining/accessing active goal representations. Furthermore, difficulties in maintaining/accessing activated goal representations could explain why low-level agents are more subject to chronic disruption and action slips in everyday behavioral situations, especially action failures involving the loss of a pursued goal (e.g., forgetting why one went to the kitchen) or a failure to encode an already achieved goal (e.g., did I close the door?). It should be noted that participants in this study had to process actions performed by other people. One could argue that such stimuli may prevent us from drawing any inference about how low-level agents process their own behaviors. However, numerous theoretical and empirical data indicate that the way people perceive others' actions depends on the same action representations involved in self-performed actions (e.g., the mirror neuron system; for a review, see Rizzolatti \& Craighero, 2004). Furthermore, it has been suggested that the action monitoring system, which allows one to guide and control an action until goal completion, may also underlie the processing of actions performed by others (Shmuelof \& Zohary, 2007).

Overall, action identification may be a key feature of the cognitive aspects of human action. One factor that is directly relevant to the clinical psychology of action is the potential for deregulation of an impaired process of action identification. From this perspective, the action identification dimension could have several implications for understanding and treating some behavioral and psychological dysfunctions. Based on reported evidence that the level of action identification may influence symptoms and mechanisms implied in various psychopathological symptoms, Watkins (2011) suggested that defective action identification may be a transdiagnostic process across psychological disorders. Indeed, the level of action identification has been implicated in several psychopathological symptoms, including obsessive-compulsive behaviors (Belayachi \& Van der Linden, 2009; Dar \& Katz, 2005), autistic traits (Marsh et al., 2010) and impulsivity (Vallacher \& Wegner, 1989). For example, people with checking symptoms have been related to a low level of action identification (Belayachi \& Van der Linden, 2009). A lack of goal identification in checking individuals could explain the diminished sense of goal completion they frequently experience and their need to repeat actions regardless of the obvious achievement of the goal. Low-level action identification has also been related to autistic traits (Marsh et al., 2010). This relationship has been interpreted as consistent with people with autism's problems interpreting and inferring other people's goals and intentions (Baron-Cohen, Leslie, \& Frith, 1986). Thus, action identification may be a key feature of both obsessive-compulsive symptoms and autism spectrum disorder. This is consistent with the idea that some overlaps exist between these two sets of symptoms (Fischer-Terworth \& Probst, 2009). Nevertheless, action identification may be differentially affected in these two groups of psychopathological symptoms: while action identification may be connected to self-regulation (i.e., self-action processing) in obsessive-compulsive symptoms, low-level action identification in people with autism spectrum may specifically concern the social domain (i.e., social cognitive processes). Action identification could also be biased toward an abstract level that is overly high, as in depression. Watkins (2011), for example, proposed that an extremely high identification of negative events may be connected to depressed mood.

In conclusion, this research focused on action identification, a dispositional tendency which is known to influence the cognitive processing of action. According to the action identification theory, low-level agents may be characterized by action representations that are predominantly based on concrete, mechanistic details and by an increased difficulty in activating/maintaining goal representations during action processing. By using the goal activation paradigm, this study provides evidence only that goal representations are less readily available in low-level agents than in high-level agents. Hence, there is still a need to explore the extent to which low-level agents, as compared to high-level agents, are also characterized by the increased activation of low-level representations (i.e., mechanistic details, instrumental aspects of action). This issue could be examined by using a modified version of the goal activation task, in which test words could refer either to the goal implied by the situation or the means and movement parameters related to that situation. By using such a task, one might expect an interaction between the level of agency and the priming of test words in the lexical decision task. Future works are also needed in order to examine the effect of action identification on action processing during on-line processing of one's own actions.

Empirical and theoretical evidences suggest that impaired action identification could be a transdiagnostic factor (Watkins, 2011), which is differentially affected across various psychopathological states. Future studies should be conducted in order to better grasp the connection between the level of action identification and certain psychological disorders. More specifically, it is advisable to not only identify the bias toward a particular level of action identification that may characterize some psychological symptoms but also specify the content domain that could be problematic (e.g., social domain in autism, personal life events in depression, and personal standards in perfectionism) (for a detailed framework for this topic, see Watkins, 2011).

\section{References}

Baron-Cohen, S., Leslie, A. M., \& Frith, U. (1986). Mechanical, behavioral and intentional understanding of picture stories in autistic children. British Journal of Developmental Psychology, 4, 113-125.

Belayachi, S., \& Van der Linden, M. (submitted for publication). Individual Differences in Cognitive Representations of Action: A French Adaptation of the Behavior Identification Form and its Relationships with Everyday Behavioral Self-regulation.

Belayachi, S., \& Van der Linden, M. (2009). Level of agency in sub-clinical checking. Consciousness and Cognition, 18, 293-299.

Csibra, G., Gergely, G., Biro, S., Koos, O., \& Brockbank, M. (1999). Goal attribution without agency cues: The perception of pure reason in infancy. Cognition, 72, 237-267.

Dar, R., \& Katz, H. (2005). Action identification in obsessive-compulsive washers. Cognitive Therapy and Research, 29, 333-341.

Fischer-Terworth, C., \& Probst, P. (2009). Obsessive-compulsive phenomena and symptoms in Asperger's disorder and high-functioning autism: An evaluative literature review. Life Span and Disability, 12, 5-27.

Hassin, R. R., Aarts, H., \& Ferguson, M. J. (2005). Automatic goal inferences. Journal of Experimental Social Psychology, 41, 129-140.

Heider, F., \& Simmel, M. (1944). An experimental study of apparent behavior. The American Journal of Psychology, 57, 243-259.

Marsh, A. A., Kozak, M. N., Wegner, D. M., Reid, M. E., Yu, H. H., \& Blair, R. J. R. (2010). The neural substrates of action identification. Social Cognitive and Affective Neuroscience, 5, 392-403. 
McKoon, G., \& Ratcliff, R. (1992). Inference during reading. Psychological Review, 99, $440-466$.

Rizzolatti, G., \& Craighero, L. (2004). The mirror neuron system. Annual Review of Neuroscience, $27,169-192$.

Shmuelof, L., \& Zohary, E. (2007). Watching others' actions: mirror representation in the parietal cortex. The Neuroscientist, 13, 667-672.

Vallacher, R. R., \& Wegner, D. M. (1985). A theory of action identification. Hillsdale, NJ: Lawrence Erlbaum Associates.

Vallacher, R. R., \& Wegner, D. M. (1987). What do people think they're doing? Action identification and human behavior. Psychological Review, 94, 3-15.
Vallacher, R. R., \& Wegner, D. M. (1989). Levels of personal agency: Individual variation in action identification. Journal of Personality and Social Psychology, 57, 660-671.

Watkins, E. (2011). Dysregulation in level of goal and action identification across psychological disorders. Clinical Psychology Review, 31, 260-278.

Wegner, D. M., Vallacher, R. R., Macomber, G., Wood, R., \& Arps, K. (1984). The emergence of action. Journal of Personality and Social Psychology, 46, 269-279.

Zacks, J. M. (2004). Using movement and intentions to understand simple events. Cognitive Science, 28, 979-1008. 